\title{
Work in Progress: Identifying Factors that Impact Student Experience of Engineering Stress Culture
}

\section{Mr. Joseph Francis Mirabelli, University of Illinois at Urbana - Champaign}

Joseph Mirabelli is an Educational Psychology graduate student at the University of Illinois at UrbanaChampaign with a focus in Engineering Education. His work focuses on mentorship, mental health, and retention in STEM students and faculty. He was awarded the NAGAP Graduate Education Research Grant award to study engineering faculty perceptions of graduate student well-being and attrition. Before studying education at UIUC, Joseph earned an MS degree in Physics from Indiana University in Bloomington and a BS in Engineering Physics at UIUC.

\section{Andrea J Kunze, University of Illinois at Urbana-Champaign}

Andrea Kunze is a PhD student at the University of Illinois at Urbana-Champaign in the Department of Educational Psychology. Prior to coming to UIUC, she completed a MS in Educational Psychology at NC State University, and a BS in Human Learning \& Development at Georgia State University. Her research currently focuses on utilizing qualitative and mixed methodologies to explore people's perceptions and experiences of the social environment in which they learn or work, and how it impacts their engagement and success.

\section{Ms. Julianna Ge, Purdue University-Main Campus, West Lafayette (College of Engineering)}

Julianna Ge is a Ph.D. student in the School of Engineering Education at Purdue University. At Purdue, she created and taught a novel course for undergraduate engineering students to explore the intersections of thriving, leadership, diversity and inclusion. As an NSF Graduate Research Fellow, her research interests intersect the fields of engineering education, positive psychology, and human development to understand diversity, inclusion, and success for undergraduate engineering students. Prior to Purdue, she received dual bachelor's degrees in Industrial Engineering and Human Development and Family Studies from the University of Illinois at Urbana-Champaign. Her prior work experiences include product management, consulting, tutoring, marketing, and information technology.

\section{Dr. Kelly J Cross, University of Nevada, Reno}

Dr. Cross is currently an Assistant Professor in the Chemical and Materials Engineering Department at the University Nevada Reno. After completing her PhD in Engineering Education at Virginia Tech in 2015, Dr. Cross worked as a post-doctoral researcher with the Illinois Foundry for Innovation in Engineering Education and in the Department of Bioengineering with the Revolutionizing Engineering Departments (RED) grant at the University of Illinois at Urbana-Champaign. Dr. Cross' scholarship investigated student teams in engineering, faculty communities of practice, and the intersectionality of multiple identity dimensions. Her research interests include diversity and inclusion in STEM, intersectionality, teamwork and communication skills, assessment, and identity construction. Her teaching philosophy focuses on student centered approaches such as culturally relevant pedagogy. Dr. Cross' complimentary professional activities promote inclusive excellence through collaboration.

\section{Prof. Karin Jensen, University of Illinois at Urbana - Champaign}

Karin Jensen, Ph.D. is a Teaching Assistant Professor in bioengineering at the University of Illinois at Urbana-Champaign. Her research interests include student mental health and wellness, engineering student career pathways, and engagement of engineering faculty in engineering education research. She was awarded a CAREER award from the National Science Foundation for her research on undergraduate mental health in engineering programs. Before joining UIUC she completed a post-doctoral fellowship at Sanofi Oncology in Cambridge, MA. She earned a bachelor's degree in biological engineering from Cornell University and a Ph.D. in biomedical engineering from the University of Virginia. 


\section{Work in Progress: Identifying Factors that Impact Student Experience of Engineering Stress Culture}

\section{Introduction}

In this work-in-progress research paper, we explore how the culture of undergraduate engineering students' departments or fields can have far-reaching effects on their success and experiences. Engineering culture has been previously described as unique compared to other disciplines, where heavy workloads and high expectations create an environment of "suffering and shared hardship" [1]. This negative culture has been described as particularly unwelcoming to women and minorities $[2,3]$ and may result in exacerbated difficulties for underrepresented groups in engineering. For these reasons, we propose that it will be critical to understand not only how students perceive this culture but also the factors that impact student experiences of engineering culture. The current work is part of a larger study to understand students' experiences within the Engineering Stress Culture (ESC) that examines student perceptions of stress as part of engineering culture through the lens of social identity theory. We have previously described correlative relationships between measures of engineering identity, inclusion, and mental health problems for engineering undergraduate students [4]. In the current work, we seek to answer the following research question: How do engineering students describe the relationship between stress and engineering culture?

Through qualitative interviews, the current project seeks to explore the characteristics of these relationships and describe how students perceive stress as a part of engineering culture. We interviewed thirty undergraduate engineering students who reported particularly high or low levels of engineering identity relative to other students in their department. The semi-structured interviews were designed based on previously conducted quantitative survey results to understand how students describe the relationships between stress, anxiety, depression, engineering identity, and inclusion. The objective of the interviews was to understand how engineering students experience stress and whether they perceive stress as part of their discipline.

The researchers asked students to define characteristics and stressors common to engineering students and professionals. In order to understand how students cope with stress, the researchers asked participants to describe coping strategies they had utilized and to assess why these strategies were healthy or unhealthy. We also asked students about their experiences using campus resources and interacting with faculty and peers on issues related to mental health. Lastly, the researchers asked students to describe stress in engineering and how stress affects their individual experience. Through inductive thematic analysis of interview transcripts, our study sought to identify factors that mediate engineering students' perceptions of identity, stress, and inclusion. Through our analyses, discipline and department-specific attributes emerged as contributors to engineering student identity, stress, and perceptions of inclusion. Further, the analyses illuminated the relationships between these dimensions and synthesized how these experiences are part of a greater ESC. Study design, data collection, and preliminary data are presented. 
Social Identity Theory The present work and the larger study is grounded in social identity theory (SIT). SIT is a broad social psychological theory that describes how one's self-concepts are related to one's membership in different social groups (e.g., occupation or gender). Further, a premise of social identity theory is that across varying contexts, different aspects of someone's social identity can become more salient [5]. SIT has been used to study a wide range of social interactions, to study both group relations and self-categorization, and to understand intergroup behaviors such as conflict, cooperation, and social change. For example, Hogg and Reid examined the role of social identity in the communication of group norms and found that group norms are not fixed, but instead can change based on the current context [6]. Further, SIT is a useful analytical tool in examining how group norms are internalized [7]. In the present work, we differentiate ingroup and outgroup members as students who have high or low levels of engineering identity. We are specifically interested in how students who identify strongly or weakly with engineering perceive norms about stress as part of the life of an engineering student. Overall, SIT provides an insightful approach to investigate the role of self-concepts and engineering identity development that may be influenced by stress or other social features of the engineering culture.

\section{Methods}

Qualitative Interview Design: The semi-structured interview protocol included 23 questions and lasted 30-60 minutes. Additional probing questions were included in the printed interview schedule. The interviews comprised four main topics: engineering identity (three questions), for example, "How would you describe a "typical" engineering student in your college and/or department?"; perceptions of stress (seven questions), for example, "Can you tell me about a time or the last time when you felt stressed?"; stress in engineering (seven questions), for example, "In your opinion, are there specific aspects of being an engineering student that are stressful?"; and coping and help-seeking behaviors (six questions), for example, "What resources and support are there on campus or in your department for students who are stressed?". Participants were asked to describe any interactions with other students and faculty regarding mental health issues and to share any other additional information about engineering-related stress. The interview protocol was developed from the results of a quantitative survey administered at the same institution in the fall of 2017, which included metrics of stress, anxiety, depression, inclusion, and engineering identity, as well as an open-ended response opportunity for participants to share additional thoughts [8]. The interview was piloted with three participants external to the participant pool. After the pilot interviews and feedback from pilot participants, some questions were re-worded for clarity, and the order of questions was adjusted.

Participants: Participants were engineering students selected from respondents to a survey in the fall of 2017. The survey indicated that if the students were willing to participate, they might be contacted for an interview at a future date. Participants were grouped as those who measure high or low engineering identity on the previous survey (upper or lower quantile compared to peers in the same department). A total of 150 students were contacted by email and offered a $\$ 30$ Amazon gift card for participation. Of the 150 contacted, 38 respondents were willing to participate (approximately 25\% response rate); however, eight could not participate due to scheduling conflicts, resulting in 30 participants. Of the 30 interview participants, 18 had measured high levels of engineering identity relative to their department peers, and 12 had 
measured low levels of engineering identity relative to their department peers. Of the 30 participants, 20 self-identified as female, 9 self-identified as male, and one participant chose not to specify a gender. Participants were enrolled as undergraduate students in one of nine departments in the college of engineering. Participants were contacted via email for interviews based on their responses to a previous survey on engineering stress culture in the fall of 2017 , and whether they indicated on the survey that they were willing to be contacted at a future date for an interview. Specifically, 150 of 1,190 students were contacted based on having either low or high engineering identity scores measured by the Identification with Academics subscale translated to engineering. Sample items include "Being good at engineering is an important part of who I am" and "It matters to me how I do in engineering school". Each item is rated on a Likert scale from (1) Strongly disagree to (7) Strongly agree [9]. Based on the information provided during the interviews, the final sample of 30 participants achieved saturation for the survey participant pool.

Data Collection: The study was approved and conducted under the institutional review board. To participate in the study, engineering students were contacted via email to schedule their interview. Interviews were conducted in the spring 2019 semester. All 30 interviews took place within a private interview room, and only the participant and one of two interviewers from the research team was present at a given time. All interviews were conducted face-to-face, audio recorded, and transcribed verbatim. The average length of the interviews was 39.1 minutes, ranging from 21 minutes to 64 minutes. All participants were assigned pseudonyms to protect participant anonymity. Pseudonyms were either selected by the participant or selected using a random generator. Participants were informed during the consent process that they could terminate the interview at any point. At the conclusion of the interview, participants were presented with a list of campus resources, including the counseling center. Interviewers completed field notes after each interview to summarize the interview and to document observations.

Data Analysis: All interviews were analyzed separately using inductive thematic analysis. This analysis used a grounded theory approach and a recommended codebook development process $[10,11]$. First, open coding was conducted on 20 of the transcribed interviews by two of the researchers. All codes were discussed by the two researchers, and then an axial coding process was used to collapse the codes based on common characteristics. Selective coding followed axial coding to determine how codes fit with the broader themes of engineering identity, stress, and coping strategies.

\section{Results}

The most significant result of the preliminary thematic analysis of the interviews is the level of stress reported by the participants. Nearly every participant suggested feeling stress often during their studies, with most of the participants reporting that the stress they experienced was constant ("I think there is always stressed [sic], always there's some level of stress"). One interview question requested participants to report a time they felt stressed, or the last time they felt stressed. Most participants reported a time within one week of the interview to describe. Participants also discussed the physical symptoms of stress ("Between me and the people I know, a lot of us have experienced hair loss."). Participants were asked to define stress and anxiety, and their definitions were inconsistent, with some participants attributing anxiety to occur on a 
shorter timescale and others attributing it to a constant experience. Participants' conceptualizations of stress, anxiety, and depression included a salient conflation of the symptoms, particularly the duration and types of physiological responses. For example, some participants' experiential definitions of anxiety included "fleeting" symptoms (e.g. a nagging sense of anxiety or a burst of worry) while others consisted of constant and gradual buildup (e.g. feeling like things were getting out of control).

Many participants acknowledged that across disciplines, undergraduate students experience high stressors from their academic work. Despite the global acknowledgement of stress, the students also reported they believe that the stress they experience as engineers is worse than the stress experienced by non-engineering students. For example, several participants suggested that the academic rigor and high ranking of the engineering department was a source of stress. Other participants recognized a competitive climate in which the high rankings fostered arrogance among peers. Participants were asked to discuss their healthy and unhealthy coping strategies, revealing a wide range of techniques used by students to cope and manage stress. Many participants divulged the use of diet in their coping mechanisms, which varied from overeating, to recreationally cooking, to focusing on better diet and exercise when feeling unwell from stress. Coping strategies considered to be unhealthy by participants included socializing (at the expense of finishing work), going to bars, playing video games, and procrastinating when feeling overwhelmed. Coping strategies considered to be healthy by the participants included goal setting, diet, exercise, engaging with hobbies, and interacting with a support system. Several participants shared experiences about university counseling and disability services, used either by participants or more often by another student close to the participants. The overall attitude of the students towards university counseling services was positive. When asked about faculty roles in the mental health culture, very few participants had interacted with faculty regarding their stressful or anxious feelings.

Participants had consistent notions regarding the characteristics of successful engineers. Participants identified traits of successful engineers in either academia or industry, including phrases such as "devoted to their careers," "hardworking," and "innovative." However, some participants identified traits in professional engineers associated more with stereotyping, such as possessing mediocre social skills. Overall, participants consistently were able to offer a selfassessment of their own shortcomings and strengths as engineering students within the context of a broader engineering identity.

\section{Discussion}

Mental health is a growing concern nationally, especially on college campuses in what has been described as a "mental health crisis" $[12,13]$. Alarming numbers of students report suffering from mental health challenges, including high levels of stress, anxiety, and depression [14-16]. To date, little research has been conducted on the mental health crisis in engineering programs, though some recent research suggests the mental health crisis is particularly acute in engineering as a discipline [17]. More research is needed on the mental health crisis within engineering programs to understand the norms in engineering culture that may perpetuate a high-stress culture. While researchers have noted high levels of mental health challenges for engineering undergraduates $[4,17]$ and documented stressors for engineering undergraduates $[8,18]$, the 
association of stress as part of engineering culture and how stress becomes normalized in engineering programs is understudied.

The preliminary interview results suggest that engineering students are undereducated in terms of mental health and available individual and institutional resources. Specifically, some participants could not identify mental health and academic counseling resources readily available in their engineering programs, suggesting a lack of accessibility. Participant responses also suggest students possess broad conceptualizations of stress, anxiety, and depression, as well as the effects of each. Conceptualizations were nuanced and reflective of clinical definitions of these terms [19]; however, the focus of our study was on experiential rather than clinical definitions.

Coping strategies for high stress levels varied largely between participants, including several participants who did not list any strategies that they considered to be healthy. However, students do have established and consistent notions of the habits of engineering students and professionals. Throughout the interviews, most participants were able to assess their healthy and unhealthy habits, knowledge, skills, and shortcomings, suggesting strong self-awareness. The healthy and unhealthy coping strategies echo prior literature regarding responses to adverse events, which ranges from thriving to succumbing to stress [20]. Within O'Leary and Ickovics' framework, people in the 'succumbing' category represent those with unhealthy coping strategies that lead to worse functioning than before experiencing the adverse event (e.g., sleeping less to study more leads to further fatigue). On the other hand, people in the thriving category represent those with healthy coping strategies that lead to better functioning than before they experienced the adverse event (e.g., exercising to alleviate stress leads to less stress).

Implications: Students can develop and promote a culture of stress within the student body by attributing stress as a group characteristic ("we are a high stress group" or "we are always stressed out - that's just the way we are") or social norm. Describing stress as a norm for the group has numerous detrimental effects on student recruitment, retention, and success. Students describing engineering or particular engineering major disciplines as "high stress" groups gives the false impression that only a certain student profile or personality is accepted, valued, or successful in engineering or specific engineering disciplines. For example, students perceive "all-nighters" to be a "rite of passage" as an engineer. The reinforcement of this behavior as a norm perpetuates a high stress work culture. This false impression risks discouraging participation or creates a barrier to student engineering identity development, which may vary by engineering disciplines, irrespective of student ability. Stress associated with engineering or attributed as being part of a culture may discourage students from seeking coping support to alleviate stress and anxiety by making them feel it is normal or even necessary for the discipline or major. Since many college students experiencing suicidal thoughts already do not seek interventions [21], at-risk engineering students may face increased risks as a result of stress culture. Perceptions of a stress culture in engineering may exacerbate individual student feelings of stress and anxiety, especially for students susceptible to anxiety disorders and those who lack social support or confidence in their abilities to perform in the program. This is particularly concerning for students who already experience higher levels of stress than their peers due to confounding and interrelated factors. In other words, stress, which is conceptually similar to microaggressions, may have cumulative effects that are not simply additive. For example, being a female student of color at a predominantly white institution has also been linked to increased 
stress levels [22], which implies that stress may impact or interact with multiple dimensions of students' identities simultaneously.

Limitations: This study has several limitations that limit the generalizability of the study. First, the study was conducted at a single institution, and the findings might vary across different institutions with different student demographics, institution size, and other variables. Additionally, the study included a limited number of underrepresented students. We invited students to participate from all departments in the college of engineering. However, not all majors were represented in the final sample due to low or no responses from students in some majors, and some major programs were overrepresented relative to overall enrollment. Since the participants were selected from respondents to an earlier survey, only students who had been enrolled for two years or longer were included in the participant pool. With this criterion, most first-year and second-year students were excluded. Another limitation of this study is that it captures the student experience at one point in time. Future work analyzing how these variables change over time will enhance our understanding of how students respond to various stressors over time and will be beneficial in building proactive interventions to improve student wellbeing. For the present studythe research team will complete an axial coding process to group the open codes based on common characteristics. Following the axial coding, the researchers will re-apply the coding scheme created from the axial process to each students' interview.

\section{Acknowledgments}

This material is based upon work supported by the National Science Foundation under Grant No. 1738186. Any opinions, findings, and conclusions or recommendations expressed in this material are those of the authors and do not necessarily reflect the views of the National Science Foundation. Advisory board members Allison Godwin, Walter Lee, and Ruby Mendenhall provided feedback on the interview protocol. The authors wish to acknowledge Nicole Jackson for scheduling and conducting interviews. Lastly, the authors wish to thank the student participants for sharing their experiences.

\section{References}

[1] E. Godfrey and L. Parker, "Mapping the cultural landscape in engineering education," Journal of Engineering Education, vol. 99, pp. 5-22, 2010.

[2] E. Godfrey, "Cultures within cultures: Welcoming or unwelcoming for women?," American Society of Engineering Education Conference Proceedings, 2007.

[3] C. E. Foor, S. E. Walden, and D. A. Trytten, "'I wish that I belonged more in this whole engineering group:" Achieving individual diversity," Journal of Engineering Education, vol. 96, pp. 103-115, 2007.

[4] K. J. Cross and K. J. Jensen, "Work in Progress: Understanding Student Perceptions of Stress as part of Engineering Culture," in American Society of Engineering Education Conference Proceedings, 2018.

[5] J. E. Stets and P. J. Burke, "Identity theory and social identity theory," Social psychology quarterly, pp. 224-237, 2000.

[6] M. A. Hogg and S. A. Reid, "Social identity, self-categorization, and the communication of group norms," Communication theory, vol. 16, pp. 7-30, 2006. 
[7] M. A. Hogg, "Social identity theory," in Understanding peace and conflict through social identity theory, ed: Springer, 2016, pp. 3-17.

[8] K. Jensen and K. J. Cross, "Board 73: Student Perceptions of Engineering Stress Culture," in 2019 ASEE Annual Conference \& Exposition, 2019.

[9] B. D. Jones, M. C. Paretti, S. F. Hein, and T. W. Knott, "An analysis of motivation constructs with first-year engineering students: Relationships among expectancies, values, achievement, and career plans," Journal of engineering education, vol. 99, pp. 319-336, 2010.

[10] Fereday, J., and E. Muir-Cochrane, Demonstrating rigor using thematic analysis: A hybrid approach of inductive and deductive coding and theme development. International Journal of Qualitative Methods, 2006. 5(1): p: 80-92.

[11] MacQueen, K. M., E. McLellan, K. Kay, and B. Milstein, B, Codebook development for team-based qualitative analysis. Cam Journal, 1996. 10(2): p. 31-36.

[12] A. Kruisselbrink Flatt, "A Suffering Generation: Six Factors Contributing to the Mental Health Crisis in North American Higher Education," College Quarterly, vol. 16, p. n1, 2013.

[13] M. Wood, "The state of mental health on college campuses," Inquiry: The Journal of the Virginia Community Colleges, vol. 17, pp. 5-15, 2012.

[14] S. K. Lipson and D. Eisenberg, "Mental health and academic attitudes and expectations in university populations: results from the healthy minds study," Journal of Mental Health, vol. 27, pp. 205-213, 2018.

[15] S. K. Lipson, E. G. Lattie, and D. Eisenberg, "Increased rates of mental health service utilization by US college students: 10-year population-level trends (2007-2017)," Psychiatric services, vol. 70, pp. 60-63, 2019.

[16] P. Brown, The Invisible Problem?: improving students' mental health: Higher Education Policy Institute Oxford, 2016.

[17] A. Danowitz and K. Beddoes, "Characterizing mental health and wellness in students across engineering disciplines," in 2018 The Collaborative Network for Engineering and Computing Diversity Conference Proceedings, 2018.

[18] L. Schneider, "Perceived stress among engineering students," in St, Lawrence Section Conference, Toronto, Canada, 2007.

[19] A. P. Association, Diagnostic and statistical manual of mental disorders (DSM-5®): American Psychiatric Pub, 2013.

[20] V. E. O'Leary and J. R. Ickovics, "Resilience and thriving in response to challenge: an opportunity for a paradigm shift in women's health," Women's health (Hillsdale, NJ), vol. 1, pp. 121-142, 1995.

[21] S. J. Garlow, J. Rosenberg, J. D. Moore, A. P. Haas, B. Koestner, H. Hendin, et al., "Depression, desperation, and suicidal ideation in college students: results from the American Foundation for Suicide Prevention College Screening Project at Emory University," Depression and anxiety, vol. 25, pp. 482-488, 2008.

[22] H. A. Neville, P. P. Heppner, P. Ji, and R. Thye, "The relations among general and racerelated stressors and psychoeducational adjustment in Black students attending predominantly White institutions," Journal of Black Studies, vol. 34, pp. 599-618, 2004. 